\title{
Improved upper bounds for planarization and series-parallelization of degree-bounded graphs
}

\author{
Keith Edwards \\ School of Computing \\ University of Dundee \\ Dundee, DD1 4HN \\ U.K. \\ kjedwards@dundee.ac.uk
}

\author{
Graham Farr \\ Clayton School of Information Technology \\ Monash University \\ Clayton, Victoria 3800 \\ Australia \\ Graham.Farr@monash.edu
}

Submitted: Jul 20, 2010; Accepted: May 23, 2012; Published: May 31, 2012

Mathematics Subject Classifications: 05C10, 05C40, 68R10, 68Q45

\begin{abstract}
We study the number of vertices which must be removed from a graph in order to make it planar or series-parallel. We give improved upper bounds on the number of vertices required to planarize graphs of bounded average degree $d$, and for small $d$ also an improved bound for series-parallelization.

The coefficient of fragmentability of a class of graphs measures the proportion of vertices that need to be removed from the graphs in the class in order to leave behind bounded sized components. The above bounds on planarization yield improved bounds for the coefficient of fragmentability of the class of connected graphs of average degree at most $d$.

As an application we give an improved bound on the size of regular expressions representing deterministic finite automata.
\end{abstract}

Keywords: fragmentability, planarization, series-parallel, tree width, regular expression.

\section{$1 \quad$ Introduction and Definitions}

In this paper we study three related parameters which measure the number of vertices which must be removed from a graph in order to make it planar, series-parallel, or fragmented (i.e. having only bounded sized components). We concentrate on bounded-degree classes of graphs.

For a graph $G$, we define $p(G)$ and $s(G)$ respectively to be the minimum size of a subset of the vertex set of $G$ whose removal leaves a graph which is, respectively, planar or series-parallel. 
The concept of fragmentability of a class of graphs was introduced by the authors in [2] and surveyed in [5]. It measures how small a proportion of vertices need to be removed from graphs in a class in order to break them into components of bounded size. We begin by recalling the definition.

Let $\varepsilon \in[0,1]$ and $C \in \mathbb{N}$. A graph $G$ is $(C, \varepsilon)$-fragmentable if there exists $X \subseteq V(G)$ such that $|X| \leqslant \varepsilon|V(G)|$ and every component of $G-X$ has at most $C$ vertices. $X$ is here called the fragmenting set. A class $\Gamma$ of graphs is $\varepsilon$-fragmentable if there exists $C \in \mathbb{N}$ such that every $G \in \Gamma$ is $(C, \varepsilon)$-fragmentable. The coefficient of fragmentability of $\Gamma$ is

$$
c_{f}(\Gamma)=\inf \{\varepsilon \mid \Gamma \text { is } \varepsilon \text {-fragmentable }\} .
$$

In [4] we showed that, if $G$ is a connected graph of average degree at most $d$, where $d \geqslant 2$, then

$$
p(G) \leqslant s(G) \leqslant \frac{d-2}{d+1}|V(G)| .
$$

It is shown in [2] that if $\Gamma$ is a class of graphs, and $p(G) \leqslant c|V(G)|+A$ for all $G \in \Gamma$, where $c, A$ are constants, then $c_{f}(\Gamma) \leqslant c$.

Let $\Gamma_{d}$ be the class of graphs with maximum degree at most $d$, and $\bar{\Gamma}_{d}^{c}$ be the class of connected graphs with average degree at most $d$. From the remarks above, and the lower bound given in [2], it follows that for $d \geqslant 2$,

$$
\frac{d-2}{2 d-2} \leqslant c_{f}\left(\bar{\Gamma}_{d}^{c}\right) \leqslant \frac{d-2}{d+1} .
$$

These results improved earlier results of Halldórsson and Lau [8] and the authors $[2,3]$.

Haxell, Pikhurko and Thomason [9] improved (for $d \geqslant 5$ ) the lower bound of (1) above by showing that, for any $d \geqslant 4$,

$$
c_{f}\left(\Gamma_{d}\right) \geqslant\left\{\begin{array}{cl}
1-\frac{4}{d+2}, & \text { if } d \text { is even; } \\
1-\frac{4(d+2)}{(d+1)(d+3)}, & \text { if } d \text { is odd. }
\end{array}\right.
$$

They also prove results on the behaviour of $c_{f}\left(\Gamma_{d}\right)$ as $d \rightarrow \infty$.

In Section 2 we improve, for any $d>3$, the upper bounds for planarization and fragmentability quoted above. We show that for a function $g(d)$, which is approximately $(d-9 / 4) /(d+1)$, we have $p(G) \leqslant g(d)|V(G)|$ for $G \in \bar{\Gamma}_{d}^{c}$. In the case of planarization we also obtain a "vertex-wise" bound, showing that $p(G) \leqslant \sum_{v \in V(G)} g(d(v))$ for any graph with minimum degree at least 3 ; thus, in effect, a vertex of degree $i$ contributes $g(i)$ towards the total number of vertices to be removed.

In Section 3 we investigate the extent to which these improved upper bounds for planarization can be extended to series-parallelization. Although the fraction $(d-2) /(d+$ $1)$ is, in general, best possible in this case, as shown by the complete graph on $d+1$ vertices, we show that for $d \leqslant 6$ this upper bound is tight for only a few small graphs, and give an 
improved upper bound $s(G) \leqslant j(d)|V(G)|+C_{d}$ for $G \in \bar{\Gamma}_{d}^{c}$, where $d \leqslant 6, j(d)<\frac{d-2}{d+1}$ and $C_{d}$ is a constant. It seems natural to expect that we will be able to extend $j(d)$ to all $d$ to obtain a corresponding vertex-wise upper bound of the form $s(G) \leqslant \sum_{v \in V(G)} j(d(v))+o(n)$ for graphs of minimum degree at least 3 (so that a vertex of degree $i$ contributes $j(i)$ to the number of vertices to be removed). However, we show that this is not possible while keeping $j(d) \leqslant \frac{d-2}{d+1}$.

The results of this paper equivalently give lower bounds on the size of the maximum induced planar (resp. series-parallel) subgraph (see for example [10] for the Maximum Induced Planar Subgraph (MIPS) problem).

We use the following notation. Let $G$ be a graph. Throughout the paper, $n=|V(G)|$ and $m=|E(G)|$. If $v \in V(G)$ then $d(v)=d_{G}(v)$ denotes the degree of $v$ in $G$.

\subsection{Reduction}

Our proofs below use the concept of series-parallel reductions. Let $G$ be a graph, and consider the following four operations on $G$ :

1. Delete an isolated vertex of $G$.

2. Delete a vertex of degree 1 (and its incident edge).

3. Let $v$ be a vertex of degree 2 with non-adjacent neighbours $x$ and $y$, delete $v$ (and edges $v x, v y$ ) and join $x$ and $y$.

4. Let $v$ be a vertex of degree 2 with adjacent neighbours, delete $v$ (and incident edges).

Let $r(G)$ be a graph obtained from $G$ by applying operations 1, 2, 3, 4 above repeatedly until none is possible (because the graph has minimum degree at least 3 ). It follows from the definition of the series-parallel property that $G$ is series-parallel if and only if $r(G)$ is empty. The construction has also been used, for example, in [1]. (The resulting graph $r(G)$ is in fact unique, but we will not need that here.) It is shown in [4] that $p(G) \leqslant p(r(G))$ and $s(G) \leqslant s(r(G))$ (in fact equality holds in each case).

\section{Planarization}

We first consider the planarization parameter $p(G)$. In order to state our upper bound, we require to define a function $g$ and list some of its properties.

For any $n \geqslant 2$, define the function $g(n)$ by setting $g(2)=0, g(3)=1 / 4$, and for any $n \geqslant 4$,

$$
n g(n)=(n-2) g(n-1)+g(n-2)+1 .
$$

Also, for any $n \geqslant 3$, define $g^{\prime}(n)=g(n)-g(n-1)$. Then it is easily established that

1. $g(n)<1$ for all $n$. This follows immediately by induction on $n$. 
2. $g$ is an increasing function of $n$. To see this we show by induction that for all $n \geqslant 3$,

$$
g(n-1) \leqslant g(n) \leqslant(1+g(n-1)) / 2
$$

This is true for $n=3$, so suppose it is true for some $n$. Then firstly,

$(n+1) g(n+1)=(n-1) g(n)+g(n-1)+1 \geqslant(n-1) g(n)+2 g(n)=(n+1) g(n)$,

and secondly

$$
(n+1) g(n+1)=(n-1) g(n)+g(n-1)+1 \leqslant n g(n)+1
$$

so that

$$
g(n+1) \leqslant \frac{n}{n+1} g(n)+\frac{1}{n+1} \leqslant \frac{1}{2} g(n)+\frac{1}{2}
$$

since $n \geqslant 1$ and $g(n)<1$.

3. $g^{\prime}$ is a decreasing function of $n$. We have

$$
\begin{aligned}
n g(n) & =(n-2) g(n-1)+g(n-2)+1 \\
(n-1) g(n-1) & =(n-3) g(n-2)+g(n-3)+1
\end{aligned}
$$

from which we obtain

$$
n g^{\prime}(n)=(n-3) g^{\prime}(n-1)+g^{\prime}(n-2) .
$$

Then it is easily shown by induction that

$$
g^{\prime}(n-1) \geqslant g^{\prime}(n) \geqslant g^{\prime}(n-1) / 3 .
$$

It is easy to see from the definition of $g$ that $n(g(n)-1)+(g(n-1)-1)$ is a constant. From this it is not hard to show that

$$
g(n)=\frac{13}{4} \frac{A(n)}{n !}+\frac{5}{4} \frac{(-1)^{n}}{n !}-\frac{9}{4}
$$

where $A(n)$ is the alternating factorial function given by $A(n)=n !-(n-1) !+\ldots-(-1)^{n} .1$.

We can now give an improved upper bound for $p(G)$. In fact we will prove something stronger. Let $W_{5}$ be the wheel on 5 vertices, and let $w_{5}(G)$ be the smallest size of a set $X \subseteq V(G)$ such that $G \backslash X$ has no $W_{5}$-minor. It is easy to see that for any graph $G$, we have $w_{5}(G)=w_{5}(r(G))$ where $r(G)$ is the reduced graph defined above.

Lemma 1. Let $G=(V, E)$ be a connected graph with $n$ vertices, regular of degree 3 . Then

$$
w_{5}(G) \leqslant(n-1) / 4 \text {. }
$$


Proof. We use induction on $n$. If $n \leqslant 5$, the result is clear, so suppose that $n>5$. For any vertex $v$, let $G^{\prime}=G-v$, and $G^{*}=r\left(G^{\prime}\right)$. Then clearly

$$
w_{5}(G) \leqslant 1+w_{5}\left(G^{\prime}\right)=w_{5}\left(G^{*}\right) .
$$

However, since deleting $v$ creates three vertices of degree 2 in $G^{\prime}$ which are removed by reduction, we have $\left|V\left(G^{*}\right)\right| \leqslant n-4$. Hence by induction, we have

$$
w_{5}(G) \leqslant 1+(n-5) / 4=(n-1) / 4
$$

as required.

Lemma 2. Let $G=(V, E)$ be a connected graph with $n$ vertices, minimum degree $\delta \geqslant 3$. Then

$$
p(G) \leqslant w_{5}(G) \leqslant \sum_{v \in V(G)} g(d(v))-g^{\prime}(\delta) .
$$

Proof. For any graph $H$, let $f(H)=\sum_{v \in V(H)} g(d(v))$, thus we need to show that $w_{5}(G) \leqslant f(G)-g^{\prime}(\delta)$.

Let $\Delta$ be the maximum degree of $G$. First, if $\Delta=3$, then $G$ is regular of degree 3 , so that $w_{5}(G) \leqslant(n-1) / 4$ from Lemma 1 above, as required. So suppose that $\Delta \geqslant 4$. We use induction on $n$. If $n<5$, there is nothing to prove, so suppose that $n \geqslant 5$.

First note that from the definition of $g$, we have, for $n \geqslant 4$,

$$
1-g(n)=(n-1) g^{\prime}(n)+g^{\prime}(n-1) .
$$

Let $w$ be a vertex of maximum degree in $G$, chosen so that the degree of its lowest degree neighbour $v_{0}$ is as small as possible. Since $G$ is connected, $d\left(v_{0}\right)<\Delta$ unless $G$ is $\Delta$-regular.

Delete the vertex $w$ to form the graph $G^{\prime}=G-w$, and let $G^{*}$ be the reduced graph $r\left(G^{\prime}\right)$. Let the components of $G^{\prime}$ be $G_{1}, \ldots, G_{k}$, with reduced graphs $G_{1}^{*}, \ldots, G_{k}^{*}$ respectively. Note that any of these reduced graphs (including $G^{*}$ ) could be empty.

By the inductive hypothesis, we have

$$
\begin{aligned}
w_{5}(G) & \leqslant 1+\sum_{i} w_{5}\left(G_{i}^{*}\right) \\
& \leqslant 1+\sum_{i} f\left(G_{i}^{*}\right)-\sum_{i} g^{\prime}\left(\delta\left(G_{i}^{*}\right)\right) \\
& =1+\sum_{i} f\left(G_{i}\right)-\sum_{i}\left(f\left(G_{i}\right)-f\left(G_{i}^{*}\right)-g^{\prime}\left(\delta\left(G_{i}^{*}\right)\right)\right) \\
& =1+f\left(G^{\prime}\right)-\sum_{i}\left(f\left(G_{i}\right)-f\left(G_{i}^{*}\right)-g^{\prime}\left(\delta\left(G_{i}^{*}\right)\right)\right),
\end{aligned}
$$

where we take $g^{\prime}\left(\delta\left(G_{i}^{*}\right)\right)$ to be zero if $G_{i}^{*}$ is empty. 
Now

$$
\begin{aligned}
1+f\left(G^{\prime}\right) & =1+f(G)-g(\Delta)-\sum_{v \sim w} g^{\prime}(d(v)) \\
& =f(G)+(\Delta-1) g^{\prime}(\Delta)+g^{\prime}(\Delta-1)-\sum_{v \sim w} g^{\prime}(d(v)) \\
& =f(G)-\sum_{v \sim w, v \neq v_{0}}\left(g^{\prime}(d(v))-g^{\prime}(\Delta)\right)-\left(g^{\prime}\left(d\left(v_{0}\right)\right)-g^{\prime}(\Delta-1)\right)
\end{aligned}
$$

Thus we have

$$
\begin{aligned}
w_{5}(G) \leqslant & f(G)-\sum_{v \sim w, v \neq v_{0}}\left(g^{\prime}(d(v))-g^{\prime}(\Delta)\right)-\left(g^{\prime}\left(d\left(v_{0}\right)\right)-g^{\prime}(\Delta-1)\right) \\
& -\sum_{i}\left(f\left(G_{i}\right)-f\left(G_{i}^{*}\right)-g^{\prime}\left(\delta\left(G_{i}^{*}\right)\right)\right) .
\end{aligned}
$$

It follows that we need to establish that in all cases, $f_{1}(G) \geqslant g^{\prime}(\delta(G))$, where

$$
f_{1}(G)=\sum_{v \sim w, v \neq v_{0}}\left(g^{\prime}(d(v))-g^{\prime}(\Delta)\right)+\left(g^{\prime}\left(d\left(v_{0}\right)\right)-g^{\prime}(\Delta-1)\right)+\sum_{i}\left(f\left(G_{i}\right)-f\left(G_{i}^{*}\right)+g^{\prime}\left(\delta\left(G_{i}^{*}\right)\right)\right) .
$$

To see this, we first note that if $G_{i}$ contains a vertex $v$ of degree $d \geqslant 3$, then

$$
f\left(G_{i}\right)-f\left(G_{i}^{*}\right)+g^{\prime}\left(\delta\left(G_{i}^{*}\right) \geqslant g^{\prime}(d) .\right.
$$

For either the vertex $v$ is still present in $G_{i}^{*}$, so that $\delta\left(G_{i}^{*}\right) \leqslant d$ and so, since $g^{\prime}$ is decreasing, $g^{\prime}\left(\delta\left(G_{i}^{*}\right)\right) \geqslant g^{\prime}(d)$, or $v$ is removed by the reduction from $G_{i}$ to $G_{i}^{*}$. But then $f\left(G_{i}\right)-f\left(G_{i}^{*}\right) \geqslant g\left(d_{G^{\prime}}(v)\right)=g(d) \geqslant g^{\prime}(d)$, as required.

If $G$ is $\Delta$-regular, then $d\left(v_{0}\right)=\Delta$, and $G^{\prime}$ will contain a vertex of degree $\Delta-1$, so that $f_{1}(G) \geqslant\left(g^{\prime}\left(d\left(v_{0}\right)\right)-g^{\prime}(\Delta-1)\right)+g^{\prime}(\Delta-1)=g^{\prime}(\Delta)$, as required.

Otherwise, we have $d\left(v_{0}\right) \leqslant \Delta-1$, so that $g^{\prime}\left(d\left(v_{0}\right)\right)-g^{\prime}(\Delta-1) \geqslant 0$. If $G^{\prime}$ contains a vertex $v$ with $\delta(G) \geqslant d_{G^{\prime}}(v) \geqslant 3$, then we have $f_{1}(G) \geqslant g^{\prime}\left(d_{G^{\prime}}(v)\right) \geqslant g^{\prime}(\delta(G))$, as required.

The only other possibility is that all neighbours of $w$ have degree 3 , and all other vertices are of degree $\Delta$. But then we have another neighbour $v_{1}$ of $w$ with degree 3 , so if some $G_{i}$ contains a vertex of degree $\Delta$, we have

$$
f_{1}(G) \geqslant\left(g^{\prime}\left(d\left(v_{1}\right)\right)-g^{\prime}(\Delta)\right)+\left(g^{\prime}\left(d\left(v_{0}\right)\right)-g^{\prime}(\Delta-1)\right)+g^{\prime}(\Delta) \geqslant g^{\prime}(3)=g^{\prime}(\delta(G)),
$$

and the result holds. Otherwise, all vertices apart from $w$ are of degree 3 and are adjacent to $w$. But then $w_{5}(G)=1$, and since $\Delta \geqslant 4, f(G) \geqslant g(4)+4 g(3) \geqslant 5 / 4$, and so $f(G)-g^{\prime}(\delta) \geqslant 1$ as required.

Corollary 3. Let $G$ be a graph, and $r(G)$ the reduced graph of $G$. Then

$$
p(G) \leqslant w_{5}(G) \leqslant \sum_{v \in V(r(G))} g\left(d_{r(G)}(v)\right) .
$$


Corollary 4. Let $d \geqslant 2$ be an integer. If $G$ is a graph with $n$ vertices and maximum degree at most $d$, then

$$
p(G) \leqslant w_{5}(G) \leqslant g(d) n .
$$

\subsection{Comparison with earlier bounds for planarization}

For $d=2,3$ this gives no improvement on previous results in [4] (since these are best possible), however for $d \geqslant 4$ we get an improved fraction compared with the previous upper bound of $\frac{d-2}{d+1} n$. To see this, recall that the function $g$ is given by:

$$
g(d)=\frac{13}{4} \frac{A(d)}{d !}+\frac{5}{4} \frac{(-1)^{d}}{d !}-\frac{9}{4} .
$$

Then since $A(d)+A(d-1)=d$ ! we have

$$
g(d)=\frac{13}{4 d !}(d !-(d-1) !+(d-2) !-A(d-3))+\frac{5}{4} \frac{(-1)^{d}}{d !}-\frac{9}{4}
$$

Since $A(d-3)<(d-3)$ !, we have

$$
g(d)=\frac{13}{4}\left(1-\frac{1}{d}+\frac{1}{d(d-1)}\right)-\frac{9}{4}+O\left(1 / d^{3}\right) .
$$

Then an easy calculation shows that

$$
g(d)=\frac{d-9 / 4}{d+1}+O\left(1 / d^{3}\right)
$$

Also, setting $h(d)=\frac{d-2}{d+1}-g(d)$, an easy calculation shows that

$$
d h(d)=(d-2) h(d-1)+h(d-2)+\frac{6}{d\left(d^{2}-1\right)} .
$$

and that $h(4)=h(5)=\frac{1}{40}$. Also note that for $K>0$, if $h(d-1) \geqslant 1 / K d$ and $h(d-2) \geqslant$ $1 / K(d-1)$, then

$$
d h(d) \geqslant \frac{d-2}{K d}+\frac{1}{K(d-1)}>\frac{d}{K(d+1)}
$$

so that $h(d) \geqslant 1 / K(d+1)$. Thus observing that $h(d) \geqslant 1 / 8(d+1)$ for $d=4,5$, we get that $g(d) \leqslant \frac{d-17 / 8}{d+1}$ for $d \geqslant 4$. Similarly $g(d) \leqslant \frac{d-11 / 5}{d+1}$ for $d \geqslant 8$ and $g(d) \leqslant \frac{d-56 / 25}{d+1}$ for $d \geqslant 18$. The first few values of $g(d)$ and $h(d)$ are as follows:

\begin{tabular}{|c|ccccccccc|}
\hline$d$ & 2 & 3 & 4 & 5 & 6 & 7 & 8 & 9 & 10 \\
\hline$\frac{d-2}{d+1}$ & 0 & $\frac{1}{4}$ & $\frac{2}{5}$ & $\frac{1}{2}$ & $\frac{4}{7}$ & $\frac{5}{8}$ & $\frac{2}{3}$ & $\frac{7}{10}$ & $\frac{8}{11}$ \\
$g(d)$ & 0 & $\frac{1}{4}$ & $\frac{3}{8}$ & $\frac{19}{40}$ & $\frac{131}{240}$ & $\frac{1009}{1680}$ & $\frac{8651}{13440}$ & $\frac{82069}{120960}$ & $\frac{855371}{1209600}$ \\
$h(d)$ & 0 & 0 & $\frac{1}{40}$ & $\frac{1}{40}$ & $\frac{43}{1680}$ & $\frac{41}{1680}$ & $\frac{103}{4480}$ & $\frac{2603}{120960}$ & $\frac{267719}{13305600}$ \\
\hline
\end{tabular}




\subsection{Bounds for average degree}

For any positive integer $k \geqslant 2$, define $f_{k}$ to be the straight line such that $f_{k}(k)=g(k)$ and $f_{k}(k+1)=g(k+1)$. Then for any real number $i$,

$$
f_{k}(i)=g(k)+(i-k) g^{\prime}(k+1) .
$$

It follows easily from the convexity of $g$ that for any non-negative integer $i \geqslant 2$,

$$
f_{k}(i) \geqslant g(i) \text {. }
$$

Also, for any $i$,

$$
\begin{aligned}
f_{k+1}(i)-f_{k}(i) & =g(k+1)+(i-k-1) g^{\prime}(k+2)-g(k)-(i-k) g^{\prime}(k+1) \\
& =(k+1-i)\left(g^{\prime}(k+1)-g^{\prime}(k+2)\right) \geqslant 0
\end{aligned}
$$

provided $i \leqslant k+1$. Hence since $f_{5}(0)=\frac{29}{240}>0$ and $f_{2}(2)=0$ it follows that $f_{k}(0)>0$ for $k \geqslant 5$ and $f_{k}(2) \geqslant 0$ for $k \geqslant 2$. Note that for any $j, f_{k}(j)-f_{k}(j-1)=g^{\prime}(k+1)$.

Proposition 5. Let $G=(V, E)$ be a graph and let $G^{\prime}=\left(V^{\prime}, E^{\prime}\right)$ be a graph obtained by applying one of the four operations above to $G$. For each $i \geqslant 0$, let $n_{i}, n_{i}^{\prime}$ be the number of vertices of degree $i$ in $G, G^{\prime}$ respectively. Let $k \geqslant 2$ be a positive integer. Then if $n_{0}=0$ or $k \geqslant 5$, we have

$$
\sum_{i \geqslant 0} f_{k}(i) n_{i}^{\prime} \leqslant \sum_{i \geqslant 0} f_{k}(i) n_{i}
$$

Proof. We consider the four operations. If $n_{0}=0$ then operation 1 is impossible. Otherwise, after operation 1 we have $n_{0}^{\prime}=n_{0}-1$, and $n_{i}^{\prime}=n_{i}$ for $i \neq 0$. Thus

$$
\sum_{i \geqslant 0} f_{k}(i) n_{i}^{\prime}=\sum_{i \geqslant 0} f_{k}(i) n_{i}-f_{k}(0) \leqslant \sum_{i \geqslant 0} f_{k}(i) n_{i}
$$

since $f_{k}(0) \geqslant 0$ for $k \geqslant 5$.

For operation 2, we delete a vertex of degree 1, adjacent to some other vertex $v$ of degree $j$ say, where $j \geqslant 1$. The degree of $v$ will change to $j-1$, hence we have

$$
\begin{aligned}
\sum_{i \geqslant 0} f_{k}(i) n_{i}^{\prime} & =\sum_{i \geqslant 0} f_{k}(i) n_{i}-f_{k}(1)-f_{k}(j)+f_{k}(j-1) \\
& =\sum_{i \geqslant 0} f_{k}(i) n_{i}-f_{k}(1)-g^{\prime}(k+1) \\
& =\sum_{i \geqslant 0} f_{k}(i) n_{i}-f_{k}(2) \\
& \leqslant \sum_{i \geqslant 0} f_{k}(i) n_{i} .
\end{aligned}
$$

For operation 3 , we have $n_{2}^{\prime}=n_{2}-1$, and $n_{i}^{\prime}=n_{i}$ for $i \neq 2$. Thus

$$
\sum_{i \geqslant 0} f_{k}(i) n_{i}^{\prime}=\sum_{i \geqslant 0} f_{k}(i) n_{i}-f_{k}(2) \leqslant \sum_{i \geqslant 0} f_{k}(i) n_{i}
$$


since $f_{k}(2) \geqslant 0$ for $k \geqslant 2$.

For operation 4 , we delete a vertex of degree 2, with two neighbours of degrees $j, j^{\prime}$ say, which both lose one neighbour. Hence

$\sum_{i \geqslant 0} f_{k}(i) n_{i}^{\prime}=\sum_{i \geqslant 0} f_{k}(i) n_{i}-f_{k}(2)-\left(f_{k}(j)-f_{k}(j-1)\right)-\left(f_{k}\left(j^{\prime}\right)-f_{k}\left(j^{\prime}-1\right)\right) \leqslant \sum_{i \geqslant 0} f_{k}(i) n_{i}$

since $f_{k}(2) \geqslant 0$ for $k \geqslant 2$ and $f_{k}(i)-f_{k}(i-1)=g^{\prime}(k) \geqslant 0$ for any $i$.

Proposition 6. Let $G=(V, E)$ be a graph. Let $r(G)$ be a reduced graph of $G$, and suppose that $r(G)$ is non-empty. For each $i \geqslant 0$, let $n_{i}, n_{i}^{\prime}$ be the number of vertices of degree $i$ in $G, r(G)$ respectively. Let $k \geqslant 2$ be a positive integer. Then if $G$ is connected or $k \geqslant 5$, we have

$$
\sum_{i \geqslant 0} f_{k}(i) n_{i}^{\prime} \leqslant \sum_{i \geqslant 0} f_{k}(i) n_{i}
$$

Proof. Let $G_{0}=G, G_{1}, \ldots, G_{k}=r(G)$ be the sequence of graphs in the reduction process. If $G$ is connected, then since $r(G)$ is non-empty, we use only operations $2,3,4$ in forming $r(G)$, and no graph in the reduction sequence has an isolated vertex. The result then follows from Proposition 5.

Lemma 7. Let $G$ be a graph, and for each $i \geqslant 0$, let $n_{i}$ be the number of vertices of degree $i$ in $G$. Let $k \geqslant 2$ be a positive integer. Then if $G$ is connected, or $k \geqslant 5$,

$$
p(G) \leqslant w_{5}(G) \leqslant \max \left\{0, \sum_{i \geqslant 0} f_{k}(i) n_{i}\right\}
$$

Proof. Let $r(G)$ be a reduced graph of $G$. If $w_{5}(G)=0$, the result follows. Otherwise, $r(G)$ is non-empty. For each $i \geqslant 0$, let $n_{i}^{\prime}$ be the number of vertices of degree $i$ in $r(G)$. Note that $n_{0}^{\prime}=n_{1}^{\prime}=n_{2}^{\prime}=0$. Then by Corollary 3 ,

$$
w_{5}(G) \leqslant \sum_{i \geqslant 3} g(i) n_{i}^{\prime}=\sum_{i \geqslant 0} g(i) n_{i}^{\prime}
$$

But as noted above, $g(i) \leqslant f_{k}(i)$ for each non-negative integer $i$, so by Proposition 6 , we have

$$
\sum_{i \geqslant 0} g(i) n_{i}^{\prime} \leqslant \sum_{i \geqslant 0} f_{k}(i) n_{i}^{\prime} \leqslant \sum_{i \geqslant 0} f_{k}(i) n_{i}
$$

as required.

Lemma 8. Let $G$ be a graph with $n$ vertices, of average degree at most $d$, where $d \geqslant 2$. Let $k \geqslant 2$ be a positive integer. Then if $G$ is connected, or $k \geqslant 5$,

$$
p(G) \leqslant w_{5}(G) \leqslant f_{k}(d) n
$$


Proof. If $w_{5}(G)=0$, then since $f_{k}(d) \geqslant 0$ when $d \geqslant 2$, the result follows. Otherwise write $f_{k}$ as $f_{k}(i)=a_{k} i+b_{k}$ where $a_{k}$ and $b_{k}$ are constants, and observe that by Lemma 7 ,

$$
\begin{aligned}
w_{5}(G) & \leqslant \sum_{i \geqslant 0} f_{k}(i) n_{i} \\
& =a_{k} \sum_{i \geqslant 0} i n_{i}+b_{k} \sum_{i \geqslant 0} n_{i} \\
& \leqslant a_{k} d n+b_{k} n \\
& =f_{k}(d) n .
\end{aligned}
$$

Taking $k=\lfloor d\rfloor$ gives the best upper bound. Let $\bar{g}(d)$ be the piecewise linear interpolant of the function $g(d)$, then $\bar{g}(d)=f_{\lfloor d\rfloor}(d)$ and we have the following:

Theorem 9. Let $G$ be a connected graph with $n$ vertices, of average degree at most $d$, where $d \geqslant 2$.

$$
\begin{aligned}
p(G) & \leqslant w_{5}(G) \leqslant \bar{g}(d) n ; \\
\text { if } d \geqslant 4, \text { then } p(G) & \leqslant w_{5}(G) \leqslant \frac{d-17 / 8}{d+1} n=\frac{d-2.125}{d+1} n ; \\
\text { if } d \geqslant 8, \text { then } p(G) & \leqslant w_{5}(G) \leqslant \frac{d-11 / 5}{d+1} n=\frac{d-2.2}{d+1} n ; \\
\text { if } d \geqslant 18, \text { then } p(G) & \leqslant w_{5}(G) \leqslant \frac{d-56 / 25}{d+1} n=\frac{d-2.24}{d+1} n .
\end{aligned}
$$

We note that since $W_{5}$ is planar, the $W_{5}$-minor-free subgraphs obtained above have bounded tree-width (in fact tree-width at most 3). We give an example of the use of this below.

\subsection{Regular expressions for finite automata}

Following the work of Gruber and Holzer [7], we can use the above bounds to give an improved bound on the size of regular expressions representing deterministic finite automata. Let $A$ be a deterministic finite automaton over an alphabet $\Sigma$, and let $L(A)$ be the language accepted by $A$. Then it is well known that $L(A)$ is a regular language which can be represented by a regular expression. The size or alphabetic width of a regular expression is the number of alphabetic symbols which it contains. Using the results of [4], Gruber and Holzer show that if $A$ has $n$ states, then it can be represented by a regular expression of size at most $|\Sigma| \cdot n^{O(1)} \cdot 4^{c \cdot n}$, where $c=(2|\Sigma|-2) /(2|\Sigma|+1$ ). (Thus for a binary input alphabet, $4^{c} \approx 1.741$.) They show this by using a transition digraph $H$ of $A$ with $n$ vertices and average (undirected) degree $d$ at most $2|\Sigma|$. By [4] there is a set $X$ of at most $\frac{2|\Sigma|-2}{2|\Sigma|+1} n$ vertices such that $H-X$ has tree-width 2 (i.e. is a series-parallel graph). Gruber and Holzer show that because $H-X$ has bounded tree-width, it contributes only a polynomial term to the size of the regular expression, while each vertex of $X$ contributes a factor of at most 4 . 
The proof given by Gruber and Holzer does not depend on $H-X$ having tree-width 2; they make clear that the same argument works provided $H-X$ has tree-width at most $k$, for any fixed $k$. Hence we can use our results above to obtain a better bound. By Theorem 9 , since $H$ has average degree at most $2|\Sigma|$, then there is a set $X$ of at most $g(2|\Sigma|) n$ vertices such that $H-X$ is $W_{5}$-minor-free, and so has tree-width at most 3 . It follows that we can replace $c=(2|\Sigma|-2) /(2|\Sigma|+1)$ in the above by $c=g(2|\Sigma|)$. Hence we have

Theorem 10. Let $A$ be a deterministic finite automaton with $n$ states over an alphabet $\Sigma$. Then the language $L(A)$ can be represented by a regular expression of alphabetic width at most $|\Sigma| \cdot n^{O(1)} \cdot 4^{g(2|\Sigma|) n}$.

In particular, for a binary input alphabet, we obtain a regular expression of size at most $n^{O(1)} \cdot 4^{(3 / 8) n}$, where $4^{3 / 8}<1.682$.

\subsection{Fragmentability}

From the above results we get the following corollary:

Corollary 11. Let $d \geqslant 2$. Let $\Gamma_{d}$ be the class of graphs with maximum degree at most $d$, and $\bar{\Gamma}_{d}^{c}$ the class of connected graphs with average degree at most $d$. Then the coefficients of fragmentability of $\Gamma_{d}$ and $\bar{\Gamma}_{d}^{c}$, i.e. $c_{f}\left(\Gamma_{d}\right)$ and $c_{f}\left(\bar{\Gamma}_{d}^{c}\right)$, are at most $g(d)$.

Proof. As mention aobve, we showed in [2] that if $\Gamma$ is a class of graphs, and $p(G) \leqslant$ $c|V(G)|+A$ for all $G \in \Gamma$, where $c, A$ are constants, then $c_{f}(\Gamma) \leqslant c$. Thus taking $\Gamma$ to be $\Gamma_{d}$ or $\bar{\Gamma}_{d}^{c}$, and setting $c=g(d)$ and $A=0$, gives the result.

Haxell et al. [9] define the constant $\gamma$ to be the limit of $d\left(1-c_{f}\left(\Gamma_{d}\right)\right)$, and show that $\frac{25}{8} \leqslant \gamma \leqslant 4$. We can improve the lower bound slightly; recall from Section 2.1 that $g(d)=\frac{d-9 / 4}{d+1}+O\left(1 / d^{3}\right)$. Then we have

$$
\begin{aligned}
d\left(1-c_{f}\left(\Gamma_{d}\right)\right) & \geqslant d(1-g(d)) \\
& =d\left(1-\frac{d-9 / 4}{d+1}-O\left(1 / d^{3}\right)\right) \\
& =d\left(\frac{13 / 4}{d+1}\right)-O\left(1 / d^{2}\right) \rightarrow \frac{13}{4} \text { as } d \rightarrow \infty .
\end{aligned}
$$

Hence $\gamma \geqslant \frac{13}{4}$.

\section{Series-parallelization}

Let $s(G)$ be the minimum number of vertices whose removal from $G$ leaves a series-parallel graph, i.e. one with no $K_{4}$-minor. It is shown in [4] that for any connected graph $G$ with average degree $d$, where $d \geqslant 2$, we have $s(G) \leqslant \frac{d-2}{d+1}|V(G)|$. In general this bound is best possible, since $K_{d+1}$ is $d$-regular, and in order to make $K_{d+1}$ series-parallel, we must remove $d-2$ vertices to leave $K_{3}$, so $s\left(K_{d+1}\right)=\frac{d-2}{d+1}\left|V\left(K_{d+1}\right)\right|$. Also, considering a $d$-regular graph on $t$ vertices ( $t$ arbitrarily large), with each vertex replaced by a copy of $K_{d}$, shows that 
there are arbitrarily large $d$-regular graphs with $s(G) \geqslant \frac{d-3}{d}|V(G)|$. However this leaves open the possibility that we can obtain an upper bound with a fraction strictly smaller than $\frac{d-2}{d+1}$, plus a smaller additive term. This is indeed true for graphs of maximum degree at most 6 , for which we can obtain an upper bound similar to that for planarization.

For any graph $G$, let $n_{i}=|\{v \mid d(v)=i\}|$. First define the function $t_{5}$ by

$$
t_{5}(G)=g(3) n_{3}+g(4) n_{4}+g(5) n_{5}=\frac{1}{4} n_{3}+\frac{3}{8} n_{4}+\frac{19}{40} n_{5} .
$$

Note that if $G$ is any graph, then in the reduced graph $r(G)$, no vertex has higher degree than in $G$, so that $t_{5}(r(G)) \leqslant t_{5}(G)$, and there are no extra components, that is, the reduction process may remove components, but never splits a component into two or more parts.

We will say that a graph is $d /(d+1)$-bi-regular if it can be constructed from two connected $d$-regular graphs $G_{1}$ and $G_{2}$ by adding an edge joining a vertex of $G_{1}$ to a vertex of $G_{2}$.

We prove the following:

Lemma 12. Let $G=(V, E)$ be a connected graph with maximum degree at most 5 and minimum degree at least 3 . Then

$$
s(G) \leqslant t_{5}(G)+a_{5}(G),
$$

where $a_{5}(G)$ is defined as follows:

$$
a_{5}(G)=\left\{\begin{array}{cl}
\frac{3}{20} & \text { if } G \text { is } 5 \text {-regular and non-empty } \\
\frac{1}{8} & \text { if } G \text { is } 4 \text {-regular and non-empty } \\
\frac{1}{20} & \text { if } G \text { is } 4 / 5 \text {-bi-regular } \\
0 & \text { otherwise. }
\end{array}\right.
$$

Proof. The proof is by induction on the number of vertices of $G$. If $G$ is empty, i.e. with 0 vertices, then we have $s(G)=t_{5}(G)=a_{5}(G)=0$ and the result follows. So suppose that $|V(G)| \geqslant 1$. If $G$ is 3-regular, then $t_{5}(G)=\frac{1}{4}|V(G)|$ and $a_{5}(G)=0$, so the result follows from [4].

So first suppose that $G$ is 2 -connected and not 3-regular. We will delete a vertex $w$ of degree 4 or 5 , such that all the neighbours of $w$ have degree (in $G$ ) at most $d(w)$, to obtain a graph $G^{\prime}$, and then form the reduced graph $G^{*}$ of $G^{\prime}$. First suppose that $w$ has degree 5 , and that $w$ has $b_{i}$ neighbours of degree $i, i=3,4,5$ (so that $b_{5}=5-b_{3}-b_{4}$ ). Write $n_{i}^{\prime}$ and $n_{i}^{*}$ for the number of vertices of degree $i$ in $G^{\prime}$ and $G^{*}$ respectively. Then $n_{3}^{\prime}=n_{3}+b_{4}-b_{3}, n_{4}^{\prime}=n_{4}+b_{5}-b_{4}=n_{4}+5-b_{3}-2 b_{4}$, and $n_{5}^{\prime}=n_{5}-b_{5}-1=n_{5}+b_{3}+b_{4}-6$.

Thus

$$
\begin{aligned}
t_{5}\left(G^{\prime}\right) & =t_{5}(G)+\frac{1}{4}\left(b_{4}-b_{3}\right)+\frac{3}{8}\left(5-b_{3}-2 b_{4}\right)+\frac{19}{40}\left(b_{3}+b_{4}-6\right) \\
& =t_{5}(G)-\frac{3}{20} b_{3}-\frac{1}{40} b_{4}-\frac{39}{40}
\end{aligned}
$$


Now $G^{*}$ will satisfy the conditions of the theorem, so by the inductive hypothesis, we have

$$
\begin{aligned}
s(G) & \leqslant 1+s\left(G^{\prime}\right) \\
& =1+s\left(G^{*}\right) \\
& \leqslant 1+t_{5}\left(G^{*}\right)+a_{5}\left(G^{*}\right) \\
& \leqslant 1+t_{5}\left(G^{\prime}\right)+a_{5}\left(G^{*}\right)-\left(t_{5}\left(G^{\prime}\right)-t_{5}\left(G^{*}\right)\right) \\
& =1+t_{5}(G)+a_{5}\left(G^{*}\right)-\left(t_{5}\left(G^{\prime}\right)-t_{5}\left(G^{*}\right)\right)-\frac{3}{20} b_{3}-\frac{1}{40} b_{4}-\frac{39}{40} \\
& =t_{5}(G)+a_{5}\left(G^{*}\right)-\left(t_{5}\left(G^{\prime}\right)-t_{5}\left(G^{*}\right)\right)-\frac{3}{20} b_{3}-\frac{1}{40} b_{4}+\frac{1}{40}
\end{aligned}
$$

If $d(w)=4$, then similar calculations show that

$$
t_{5}\left(G^{\prime}\right)=t_{5}(G)-\frac{1}{8} b_{3}-\frac{7}{8}
$$

and

$$
s(G) \leqslant t_{5}(G)+a_{5}\left(G^{*}\right)-\left(t_{5}\left(G^{\prime}\right)-t_{5}\left(G^{*}\right)\right)-\frac{1}{8} b_{3}+\frac{1}{8} .
$$

Now if some vertex is removed by the reduction process, i.e. there is a vertex of degree at least 3 in $G^{\prime}$ which is not present in $G^{*}$, then $t_{5}\left(G^{*}\right) \leqslant t_{5}\left(G^{\prime}\right)-\frac{1}{4}$. Then if $d(w)=5$, we have

$$
s(G) \leqslant t_{5}(G)+a_{5}\left(G^{*}\right)-\frac{1}{4}+\frac{1}{40}<t_{5}(G)
$$

since $a_{5}\left(G^{*}\right) \leqslant \frac{3}{20}$ in all cases. Similarly if $d(w)=4$, then we have

$$
s(G) \leqslant t_{5}(G)+a_{5}\left(G^{*}\right)-\frac{1}{4}+\frac{1}{8}<t_{5}(G)
$$

since $G^{*}$ has no vertex of degree 5 and so $a_{5}\left(G^{*}\right) \leqslant \frac{1}{8}$.

Hence we can assume that no vertex of $G^{\prime}$ of degree at least 3 is removed by reduction (though it is possible that reduction may reduce the degree of some vertices).

If $G$ is $K_{6}$, then a simple calculation shows that the theorem holds. If $G$ is 5 -regular, but $G \neq K_{6}$, then we have $d(w)=5$ and $b_{3}=b_{4}=0$; also, $G^{*}$ cannot be 4-regular, 5-regular, or 4/5-bi-regular, so $a_{5}\left(G^{*}\right)=0$. Hence

$$
s(G) \leqslant t_{5}(G)+\frac{1}{40}
$$

as required.

If $G$ is 4-regular, then $d(w)=4$, and $G^{*}$ has vertices of degree 3 so $a_{5}\left(G^{*}\right)=0$. Hence

$$
s(G) \leqslant t_{5}(G)+\frac{1}{8}
$$

as required.

Since we are assuming that $G$ is 2 -connected, $G$ cannot be $4 / 5$-bi-regular.

If $G$ is not 5-regular, 4-regular, or 4/5-bi-regular, then since $G$ is connected, we can choose $w$ so that $w$ has a neighbour of degree strictly less than $d(w)$. If $d(w)=5$, then $b_{3}+b_{4} \geqslant 1$. If $b_{3} \geqslant 1$, we have

$$
\begin{aligned}
s(G) & \leqslant t_{5}(G)+a_{5}\left(G^{*}\right)-\left(t_{5}\left(G^{\prime}\right)-t_{5}\left(G^{*}\right)\right)-\frac{3}{20} b_{3}-\frac{1}{40} b_{4}+\frac{1}{40} \\
& \leqslant t_{5}(G)+a_{5}\left(G^{*}\right)-\frac{3}{20} b_{3}-\frac{1}{40} b_{4}+\frac{1}{40} \\
& \leqslant t_{5}(G)+a_{5}\left(G^{*}\right)-\frac{1}{40} b_{4}+\frac{1}{8} .
\end{aligned}
$$


If $b_{3}<5$, then $G^{\prime}$ has a vertex of degree at most 3 or 4 , hence so does $G^{*}$ (since no vertices of degrees 3 and 4 are removed by reduction) and so $a_{5}\left(G^{*}\right) \leqslant \frac{1}{8}$ and the result follows. If $b_{3}=5$, then $s(G)<t_{5}(G)$ whatever value $a_{5}\left(G^{*}\right)$ has.

If $b_{3}=0$, so $b_{4} \geqslant 1$, then no reduction takes place, so $G^{*}$ has a vertex of degree 3 and so $a_{5}\left(G^{*}\right)=0$ and $s(G) \leqslant t_{5}(G)$ as required.

If $d(w)=4$, then we have $b_{3} \geqslant 1$ and

$$
s(G) \leqslant t_{5}(G)+a_{5}\left(G^{*}\right)-\frac{1}{8} b_{3}+\frac{1}{8} .
$$

If $b_{3}=4$, then $s(G)<t_{5}(G)$ whatever value $a_{5}\left(G^{*}\right)$ has. If $b_{3} \leqslant 3$, then $G^{\prime}$ and therefore $G^{*}$ has a vertex of degree 3 , so $a_{5}\left(G^{*}\right)=0$, and so $s(G) \leqslant t_{5}(G)$.

Now we can assume that $G$ is not 2-connected. Choose a cutvertex $w$ of least degree. We will split $w$ into two vertices $w_{1}$ and $w_{2}$, so that each neighbour of $w$ is adjacent to exactly one of $w_{1}$ and $w_{2}$, and $d\left(w_{1}\right) \leqslant d\left(w_{2}\right)$. We will always be able to do this so that $d\left(w_{1}\right) \leqslant 2$ (and so will be removed by reduction). Let the two components formed by the split be $G_{1}$ and $G_{2}$.

We observe that

$$
\begin{aligned}
s(G) \leqslant & s\left(G_{1}\right)+s\left(G_{2}\right) \\
= & s\left(G_{1}^{*}\right)+s\left(G_{2}^{*}\right) \\
\leqslant & t_{5}\left(G_{1}^{*}\right)+t_{5}\left(G_{2}^{*}\right)+a_{5}\left(G_{1}^{*}\right)+a_{5}\left(G_{2}^{*}\right) \\
\leqslant & t_{5}\left(G_{1}\right)+t_{5}\left(G_{2}\right)+a_{5}\left(G_{1}^{*}\right)+a_{5}\left(G_{2}^{*}\right)-\left(t_{5}\left(G_{1}\right)-t_{5}\left(G_{1}^{*}\right)\right)-\left(t_{5}\left(G_{2}\right)-t_{5}\left(G_{2}^{*}\right)\right) \\
\leqslant & t_{5}(G)+a_{5}\left(G_{1}^{*}\right)+a_{5}\left(G_{2}^{*}\right)-\left(g(d(w))-g\left(d\left(w_{1}\right)\right)-g\left(d\left(w_{2}\right)\right)\right) \\
\quad & \quad-\left(t_{5}\left(G_{1}\right)-t_{5}\left(G_{1}^{*}\right)\right)-\left(t_{5}\left(G_{2}\right)-t_{5}\left(G_{2}^{*}\right)\right) .
\end{aligned}
$$

If $d(w)=3$, then we have $d\left(w_{1}\right)=1$ and $d\left(w_{2}\right)=2$. Reduction of $w_{1}$ will reduce the degree of its neighbour, so $t_{5}\left(G_{1}\right)-t_{5}\left(G_{1}^{*}\right) \geqslant g(5)-g(4)=\frac{1}{10}$. Hence

$$
\begin{aligned}
s(G) & \leqslant t_{5}(G)+a_{5}\left(G_{1}^{*}\right)+a_{5}\left(G_{2}^{*}\right)-\frac{1}{4}-\frac{1}{10} \\
& =t_{5}(G)+a_{5}\left(G_{1}^{*}\right)+a_{5}\left(G_{2}^{*}\right)-\frac{7}{20} \\
& <t_{5}(G)
\end{aligned}
$$

since $a_{5}\left(G_{1}^{*}\right)+a_{5}\left(G_{2}^{*}\right) \leqslant \frac{3}{10}<\frac{7}{20}$.

If $d(w)=4$, suppose that $d\left(w_{1}\right)=1$ and $d\left(w_{2}\right)=3$. Then as above, we have

$$
\begin{aligned}
s(G) & \leqslant t_{5}(G)+a_{5}\left(G_{1}^{*}\right)+a_{5}\left(G_{2}^{*}\right)-\left(\frac{3}{8}-\frac{1}{4}\right)-\frac{1}{10} \\
& =t_{5}(G)+a_{5}\left(G_{1}^{*}\right)+a_{5}\left(G_{2}^{*}\right)-\frac{9}{40} \\
& <t_{5}(G)
\end{aligned}
$$

since $a_{5}\left(G_{1}^{*}\right) \leqslant \frac{3}{20}<\frac{9}{40}$ and $a_{5}\left(G_{2}^{*}\right)=0$.

Now suppose that $d\left(w_{1}\right)=d\left(w_{2}\right)=2$. Then

$$
\begin{aligned}
s(G) & \leqslant t_{5}(G)+a_{5}\left(G_{1}^{*}\right)+a_{5}\left(G_{2}^{*}\right)-\frac{3}{8} \\
& <t_{5}(G)
\end{aligned}
$$


since $a_{5}\left(G_{1}^{*}\right)+a_{5}\left(G_{2}^{*}\right) \leqslant \frac{3}{10}<\frac{3}{8}$.

If $d(w)=5$, suppose that $d\left(w_{1}\right)=2$ and $d\left(w_{2}\right)=3$. Then

$$
\begin{aligned}
s(G) & \leqslant t_{5}(G)+a_{5}\left(G_{1}^{*}\right)+a_{5}\left(G_{2}^{*}\right)-\left(\frac{19}{40}-\frac{1}{4}\right) \\
& =t_{5}(G)+a_{5}\left(G_{1}^{*}\right)+a_{5}\left(G_{2}^{*}\right)-\frac{9}{40} \\
& <t_{5}(G)
\end{aligned}
$$

since $a_{5}\left(G_{1}^{*}\right) \leqslant \frac{3}{20}<\frac{9}{40}$ and $a_{5}\left(G_{2}^{*}\right)=0$. have

Finally suppose that $d\left(w_{1}\right)=1$ and $d\left(w_{2}\right)=4$. Then $t_{5}\left(G_{1}\right)-t_{5}\left(G_{1}^{*}\right) \geqslant \frac{1}{10}$. So we

$$
\begin{aligned}
s(G) & \leqslant t_{5}(G)+a_{5}\left(G_{1}^{*}\right)+a_{5}\left(G_{2}^{*}\right)-\left(\frac{19}{40}-\frac{3}{8}\right)-\frac{1}{10} \\
& =t_{5}(G)+a_{5}\left(G_{1}^{*}\right)+a_{5}\left(G_{2}^{*}\right)-\frac{1}{5} .
\end{aligned}
$$

Now $G_{1}^{*}$ and $G_{2}^{*}$ cannot be 5-regular. If at most one is 4-regular, then $a_{5}\left(G_{1}^{*}\right)+a_{5}\left(G_{2}^{*}\right) \leqslant$ $\frac{1}{8}+\frac{1}{20}<\frac{1}{5}$, so $s(G) \leqslant t_{5}(G)$ as required. If both are 4-regular, then $a_{5}\left(G_{1}^{*}\right)+a_{5}\left(G_{2}^{*}\right)=\frac{1}{4}$, and $G$ is $4 / 5$-bi-regular (note that the neighbour of $w_{1}$ cannot be of degree 3 since $w$ is assumed to a cutvertex of minimum degree), so that

$$
s(G) \leqslant t_{5}(G)+\frac{1}{4}-\frac{1}{5}=t_{5}(G)+\frac{1}{20}
$$

as required.

It is not possible to extend Lemma 12 to graphs of maximum degree 6 using the function $g$, because the example of a number of copies of $K_{6}$ joined cyclically by extra edges shows that $s(G)-\sum_{d=3}^{6} g(d) n_{d}$ can be arbitrarily large. However a similar result can be proved with $g(6)$ replaced by $11 / 20$ (which is still smaller than $(d-2) /(d+1)$ ).

Set $j(d)=g(d)$ for $d \leqslant 5$, and $j(6)=11 / 20$.

Define the function $t_{6}$ by

$$
t_{6}(G)=\sum_{d=3}^{6} j(d) n_{d}=\frac{1}{4} n_{3}+\frac{3}{8} n_{4}+\frac{19}{40} n_{5}+\frac{11}{20} n_{6} .
$$

Lemma 13. Let $G=(V, E)$ be a connected graph with maximum degree at most 6 and minimum degree at least 3 . Then

$$
s(G) \leqslant t_{6}(G)+a_{6}(G)
$$

where $a_{6}(G)$ is defined as follows:

$$
a_{6}(G)=\left\{\begin{array}{cl}
\frac{3}{20} & \text { if } G \text { has no vertex of degree } 3 \\
0 & \text { otherwise. }
\end{array}\right.
$$

Proof. As above, the proof is by induction on the number of vertices of $G$. If $G$ is empty, i.e. with 0 vertices, then we have $s(G)=t_{6}(G)=a_{6}(G)=0$ and the result follows. 
So suppose that $|V(G)| \geqslant 1$. If $G$ has maximum degree at most 5 , the result follows from Lemma 12. So suppose that $G$ has a vertex of degree 6 .

If $G$ has a vertex $w$ of degree 6 which is not a cut-vertex, then delete $w$ to obtain a connected graph $G^{\prime}$, and then form the reduced graph $G^{*}$ of $G^{\prime}$.

Now $G^{*}$ will satisfy the conditions of the lemma, so by the inductive hypothesis,

$$
\begin{aligned}
s(G) & \leqslant 1+s\left(G^{\prime}\right) \\
& =1+s\left(G^{*}\right) \\
& \leqslant 1+t_{6}\left(G^{*}\right)+a_{6}\left(G^{*}\right) \\
& \leqslant 1+t_{6}\left(G^{\prime}\right)+a_{6}\left(G^{*}\right)-\left(t_{6}\left(G^{\prime}\right)-t_{6}\left(G^{*}\right)\right) \\
& =1+t_{6}(G)+a_{6}\left(G^{*}\right)-\left(t_{6}\left(G^{\prime}\right)-t_{6}\left(G^{*}\right)\right)-j(6)-\sum_{v \sim w}(j(d(v))-j(d(v)-1)) .
\end{aligned}
$$

But

$$
j(6)+\sum_{v \sim w}(j(d(v))-j(d(v)-1)) \geqslant \frac{11}{20}+6\left(\frac{11}{20}-\frac{19}{40}\right)=1 .
$$

Hence since $a_{6}\left(G^{*}\right) \leqslant \frac{3}{20}$, the result holds if $G$ has no vertex of degree 3 . If $G$ has a vertex of degree 3 adjacent to $w$, then

$$
j(6)+\sum_{v \sim w}(j(d(v))-j(d(v)-1)) \geqslant \frac{11}{20}+5\left(\frac{11}{20}-\frac{19}{40}\right)+\frac{1}{4}=\frac{47}{40}>1+\frac{3}{20}
$$

and again the result follows. Finally if there is a vertex of degree 3 not adjacent to $w$, then either $G^{*}$ has a vertex of degree 3 and so $a_{6}\left(G^{*}\right)=0$, or $t_{6}\left(G^{\prime}\right)-t_{6}\left(G^{*}\right) \geqslant j(3)=\frac{1}{4}$, and in either case we get $s(G) \leqslant t_{6}(G)$.

We can now assume that $G$ has a cutvertex of degree 6 . Suppose that $w$ is a cutvertex (of any degree). As in the proof of Lemma 12, this can be split into two vertices $w_{1}$ and $w_{2}$ with $d\left(w_{1}\right) \leqslant d\left(w_{2}\right)$. Let the two components formed by the split be $G_{1}$ and $G_{2}$.

As in the proof of Lemma 12, we observe that

$$
\begin{gathered}
s(G) \leqslant t_{6}(G)+a_{6}\left(G_{1}^{*}\right)+a_{6}\left(G_{2}^{*}\right)-\left(g(d(w))-g\left(d\left(w_{1}\right)\right)-g\left(d\left(w_{2}\right)\right)\right) \\
-\left(t_{6}\left(G_{1}\right)-t_{6}\left(G_{1}^{*}\right)\right)-\left(t_{6}\left(G_{2}\right)-t_{6}\left(G_{2}^{*}\right)\right) .
\end{gathered}
$$

First suppose that $G$ has a cutvertex of degree 3 , so that $d\left(w_{1}\right)=1$ and $d\left(w_{2}\right)=2$. Then $g(d(w))-g\left(d\left(w_{1}\right)\right)-g\left(d\left(w_{2}\right)\right)=\frac{1}{4}$, and $t_{6}\left(G_{1}\right)-t_{6}\left(G_{1}^{*}\right) \geqslant \frac{11}{20}-\frac{19}{40}=\frac{3}{40}$, hence

$$
\begin{aligned}
s(G) & \leqslant t_{6}(G)+a_{6}\left(G_{1}^{*}\right)+a_{6}\left(G_{2}^{*}\right)-\frac{13}{40} \\
& <t_{6}(G) .
\end{aligned}
$$

Now suppose that there is no cutvertex of degree 3. We know there is a cutvertex of degree 6 , so suppose that $d\left(w_{1}\right)=d\left(w_{2}\right)=3$. Then $a_{6}\left(G_{1}^{*}\right)=a_{6}\left(G_{2}^{*}\right)=0$, so

$$
\begin{aligned}
s(G) & \leqslant t_{6}(G)-\left(\frac{11}{20}-2\left(\frac{1}{4}\right)\right) \\
& <t_{6}(G)
\end{aligned}
$$

as required. 
Now suppose that $d\left(w_{1}\right)=2$ and $d\left(w_{2}\right)=4$. If $G$ contains a vertex of degree 3 , then so does $G_{i}$ say. Then either $G_{i}^{*}$ has a vertex of degree 3 and so $a_{6}\left(G_{i}^{*}\right)=0$, or $t_{6}\left(G_{i}\right)-t_{6}\left(G_{i}^{*}\right) \geqslant \frac{1}{4}$, and in either case we have $a_{6}\left(G_{i}^{*}\right)-\left(t_{6}\left(G_{i}\right)-t_{6}\left(G_{i}^{*}\right)\right) \leqslant 0$. Therefore

$$
\begin{aligned}
s(G) & \leqslant t_{6}(G)+\frac{3}{20}-\left(\frac{11}{20}-\frac{3}{8}\right) \\
& <t_{6}(G)
\end{aligned}
$$

as required. If $G$ has no vertex of degree 3, then

$$
\begin{aligned}
s(G) & \leqslant t_{6}(G)+2\left(\frac{3}{20}\right)-\left(\frac{11}{20}-\frac{3}{8}\right) \\
& <t_{6}(G)+\frac{3}{20} .
\end{aligned}
$$

Finally, if $d\left(w_{1}\right)=1$ and $d\left(w_{2}\right)=5$, then $w_{1}$ has a single neighbour in $G_{1}$ which cannot be of degree 3 (for then that vertex would be a cutvertex of degree 3 in $G$ ). Let $G_{1}^{\prime}$ be the graph which results from deleting $w_{1}$ from $G_{1}$. Then we have

$$
\begin{aligned}
s(G) \leqslant t_{6}(G)+a_{6}\left(G_{1}^{*}\right)+a_{6}\left(G_{2}^{*}\right)-\left(g(d(w))-g\left(d\left(w_{1}\right)\right)-g\left(d\left(w_{2}\right)\right)\right) \\
-\left(t_{6}\left(G_{1}\right)-t_{6}\left(G_{1}^{\prime}\right)\right)-\left(t_{6}\left(G_{1}^{\prime}\right)-t_{6}\left(G_{1}^{*}\right)\right)-\left(t_{6}\left(G_{2}\right)-t_{6}\left(G_{2}^{*}\right)\right) .
\end{aligned}
$$

Now $t_{6}\left(G_{1}\right)-t_{6}\left(G_{1}^{\prime}\right) \geqslant \frac{3}{40}$, and $g(d(w))-g\left(d\left(w_{1}\right)\right)-g\left(d\left(w_{2}\right)\right)=\frac{3}{40}$ also.

Thus

$$
s(G) \leqslant t_{6}(G)+a_{6}\left(G_{1}^{*}\right)+a_{6}\left(G_{2}^{*}\right)-\frac{3}{20}-\left(t_{6}\left(G_{1}^{\prime}\right)-t_{6}\left(G_{1}^{*}\right)\right)-\left(t_{6}\left(G_{2}\right)-t_{6}\left(G_{2}^{*}\right)\right) .
$$

If either of $G_{1}^{\prime}$ or $G_{2}$ has a vertex of degree 3 , then, as above, we have either $a_{6}\left(G_{1}^{*}\right)-$ $\left(t_{6}\left(G_{1}^{\prime}\right)-t_{6}\left(G_{1}^{*}\right)\right) \leqslant 0$ or $a_{6}\left(G_{2}^{*}\right)-\left(t_{6}\left(G_{2}\right)-t_{6}\left(G_{2}^{*}\right)\right) \leqslant 0$, and so $s(G) \leqslant t_{6}(G)$. Otherwise, $G$ has no vertex of degree 3 , and we have $s(G) \leqslant t_{6}(G)+\frac{3}{20}$ as required.

We summarise these results in the following:

Theorem 14. Let $G=(V, E)$ be a connected graph with maximum degree at most 6 . For each $i$ let $n_{i}$ be the number of vertices with degree $i$. Then

$$
s(G) \leqslant \frac{1}{4} n_{3}+\frac{3}{8} n_{4}+\frac{19}{40} n_{5}+\frac{11}{20} n_{6}+\frac{3}{20} .
$$

It would seem natural to attempt to extend the function $j$ to values of $d \geqslant 7$, and hope to show that for any connected graph with maximum degree $\Delta$ and minimum degree at least $3, s(G) \leqslant \sum_{d=3}^{\Delta} j(d) n_{d}+C_{\Delta}$, where $C_{\Delta}$ is a constant depending on $\Delta$. However, even for $\Delta=8$, it is not possible to do this while keeping $j(d) \leqslant \frac{d-2}{d+1}$ for each $d$, which is desirable since we know that $s(G) \leqslant \sum_{d=3}^{\Delta} \frac{d-2}{d+1} n_{d}$. For consider a graph $G_{d}(t)$ as follows: take $t$ copies of $K_{d-1}$, each with one vertex distinguished, and join these distinguished vertices in a $t$-cycle, so $G_{d}(t)$ has maximum degree $d$. Then for $G=G_{8}(t)$, we have (provided $\left.j(8) \leqslant \frac{8-2}{8+1}=\frac{2}{3}\right) s(G)-\sum_{d=3}^{8} j(d) n_{d} \geqslant t / 30$ which is unbounded.

We might also hope, as in the planarization case, to show that there is a constant $\varepsilon>0$ such that for graphs of maximum degree at most $d, s(G) \leqslant \frac{d-2-\varepsilon}{d+1}|V(G)|+C_{d}$, 
where $C_{d}$ is a constant. But again the example of $G_{d}(t)$ above shows that for large enough $d, s(G)-\frac{d-2-\varepsilon}{d+1}|V(G)|$ will be unbounded. It is also easy to construct similar $d$-regular examples.

Finally, it is worth pointing out that planarization is genuinely easier than seriesparallelization, at least for high enough degree. For any class $\Gamma$, we can define constants $c_{p}(\Gamma), c_{s}(\Gamma)$ as follow:

$$
c_{p}(\Gamma)=\min \{\lambda: p(G) \leqslant(\lambda+o(1))|V(G)| \text { for all } G \in \Gamma\}
$$

and

$$
c_{s}(\Gamma)=\min \{\lambda: s(G) \leqslant(\lambda+o(1))|V(G)| \text { for all } G \in \Gamma\} .
$$

(Note that it is easy to see that these really are minima rather than just infima.) Then $c_{p}\left(\Gamma_{d}^{c}\right)$ and $c_{s}\left(\Gamma_{d}^{c}\right)$ are, respectively, the smallest proportions of the vertices which will planarize or series-parallelize connected graphs of maximum degree $d$. We know, of course, that $c_{p}\left(\Gamma_{3}^{c}\right)=c_{s}\left(\Gamma_{3}^{c}\right)=1 / 4$. However, using the example above of a $d$-regular graph on $t$ vertices ( $t$ arbitrarily large), with each vertex replaced by a copy of $K_{d}$, it is easy to see that $c_{s}\left(\Gamma_{d}^{c}\right) \geqslant(d-3) / d$. Then, for $d \geqslant 13$, we have

$$
c_{p}\left(\Gamma_{d}^{c}\right) \leqslant g(d)<(d-3) / d \leqslant c_{s}\left(\Gamma_{d}^{c}\right)
$$

so planarization requires a strictly smaller proportion of the vertices to be removed.

\section{References}

[1] H. de Fraysseix and P. Ossona de Mendez, A characterization of DFS cotree critical graphs, in: P. Mutzel, M. Jünger and S. Leipert (eds.), Graph Drawing 2001 (Vienna, 23-26 Sept. 2001), Lecture Notes in Computer Science 2265, Springer, Berlin, 2002, pp. 84-95.

[2] K. J. Edwards and G. E. Farr, Fragmentability of graphs, J. Combin. Theory (Ser. B) 82 (2001) 30-37.

[3] K. J. Edwards and G. E. Farr, An algorithm for finding large induced planar subgraphs, in: P. Mutzel, M. Jünger and S. Leipert (eds.), Graph Drawing 2001 (Vienna, 23-26 Sept. 2001), Lecture Notes in Computer Science 2265, Springer, Berlin, 2002, pp. $75-83$.

[4] K. J. Edwards and G. E. Farr, Planarization and fragmentability of some classes of graphs, Discrete Math. 308 (2008) 2396-2406.

[5] K. J. Edwards and G. E. Farr, Graph fragmentability, in: L. W. Beineke and R. J. Wilson (eds.), Topics in Structural Graph Theory, Cambridge University Press, to appear.

[6] K. J. Edwards and C. J. H. McDiarmid, New upper bounds on harmonious colorings, J. Graph Theory 18 (1994) 257-267. 
[7] H. Gruber and M. Holzer, Provably Shorter Regular Expressions from Deterministic Finite Automata (Extended Abstract), in: M. Ito and M. Toyama (eds.), Proceedings of the 12th International Conference Developments in Language Theory (Kyoto, Japan, September 2008), Lecture Notes in Computer Science 5257, Springer, Berlin, 2008, pp. 383-395.

[8] M. M. Halldórsson and H. C. Lau, Low-degree graph partitioning via local search with applications to constraint satisfaction, max cut, and coloring, J. Graph Algorithms Appl. 1 (3) (1997) 1-13.

[9] P. Haxell, O. Pikhurko and A. Thomason, Maximum acyclic and fragmented sets in regular graphs, J. Graph Theory 57 (2) (2008) 149-156.

[10] A. Liebers, Planarizing graphs - a survey and annotated bibliography, J. Graph Algorithms Appl. 5 (1) (2001) 1-74. 